\title{
WILEY-VCH
}

\section{Application of Multifunctional Mechanical Metamaterials}

Ali Shanian, Francois- Xavier Jette, Mahtab Salehii, Minh Quan Pham, Megan Schaenzer, Genevieve Bourgeois, Katia Bertoldi, Andrew Gross, Farhad Javid, David Backman*, Scott Yandt*, Miklos Gerendas, Tobias Meis, Karsten Knobloch, Friedrich Bake, and Dieter Peitsch

Dr. A. Shanian, Dr. F. Jette, Dr. M. Salehii, M.Q. Pham, M. Schaenzer, G. Bourgeois Siemens Canada, 9545 Chemin de la Cote-de-Liesse, Dorval, QC, H9P 1A5, Canada

Prof. K. Bertoldi, Dr. A. J. Gross, Dr. F. Javid

Harvard University, Cambridge, MA 02138, USA

Dr. D. Backman, Dr. S. Yandt

National Research Council Canada, 1200 Montreal Road, K1A 0R6 Canada

david.backman@nrc-cnrc.gc.ca

Dr. S. Yandt

National Research Council Canada, 1200 Montreal Road, K1A 0R6 Canada

scott.yandt@nrc-cnrc.gc.ca

Dr.-Ing. M. Gerendas

Rolls-Royce Deutschland, Eschenweg 11, Dahlewitz 15827 Blankenfelde-Mahlow, Germany

Dipl-Ing. T. Meis

Siemens AG, Huttenstr. 12, 10553 Berlin, Germany

Dr-Ing. K. Knobloch, Dr-Ing. F. Bake

German Aerospace Center (DLR), Triebwerksakustik, Mueller-Breslau-Str. 8, 10623 Berlin, Germany

Prof. D. Peitsch

TU Berlin, Marchstrasse 12-14, 105807 Berlin, Germany

Keywords: architected material, negative poisson's ratio, thermomechanical fatigue, film cooling, hot acoustic damping

Abstract

Mechanical metamaterials have attracted great interest due their ability to attain material properties outside the bounds of those found in natural materials. Many promising mechanical metamaterials have been designed, fabricated, and tested, however these metamaterials have not been subjected to the rigorous requirements needed to certify their use in demanding 


\section{WILEY-VCH}

industrial applications that require multifunctional behavior. This paper details an auxetic multifunctional metamaterial that has been optimized to outperform conventional designs for cooling systems commonly used in the space, the transportation, the energy and the nuclear industry. Experimental testing performed to certify this material for use in gas turbines have shown that in comparison to conventional designs, the metamaterial increases structural life by orders of magnitude while also providing more efficient cooling and maintaining the acoustic damping characteristics, while requiring solutions offers an agile and economical solution for the realization of next generation components

Interest in meta-materials has been soaring since their conception nearly twenty years ago. ${ }^{[1]}$ Their unique properties, derived from form rather than composition, and clear opportunities for application in the domain of electromagnetics has resulted in quick translation from conception to commercial application. ${ }^{[2,3,4]}$ Their mechanical counterparts have also gathered substantial interest and have shown exotic properties such as negative

effective mass, acoustic band gaps, negative Poisson's ratio, bi-stability, among others. ${ }^{[5,6,7]}$ However, although many interesting properties have been demonstrated by mechanical metamaterials, the progression to commercial application has been a barrier, as commercial usage places substantial additional demands on a system.

Some of the most demanding applications of modern engineering are for components exposed to high temperatures and other extreme operating conditions, such as components in gas turbine engines, ${ }^{[8]}$ which must meet stringent requirements to achieve a reasonable allowed-time-in-service while producing competitive levels of thermal efficiency. As a result, the hot section components in modern gas turbine engines such as turbine blades and combustor are typically exposed to gas temperatures up to $1500^{\circ} \mathrm{C}$ and pressures up to $3 \mathrm{MPa}$ under continuous operating conditions. These extreme thermal conditions require various 


\section{WILEY-VCH}

cooling systems to be integrated into the components, such as the effusive cooling holes shown on a combustor in Figure 1, to prevent over heating of the materials. ${ }^{[8]}$ Although necessary for high temperature service, these cooling systems tend to come with an adverse effect on the mechanical integrity of the components by adding stress concentrating features and additional thermal stresses which accelerate the initiation of fatigue cracking. Improvement of these components is conventionally achieved through the development of superior materials that can endure higher temperatures and are more resistant to fatigue cracking. This method of improvement requires immense capital investment with long design cycles, retooling of manufacturing facilities, and supply chain modifications. More efficient approaches to bring next generation products to market are highly desirable.

Here, we show that a mechanical metamaterial consisting of a thin sheet with an embedded array of shaped holes ${ }^{[9]}$ can significantly improve the performance of the combustor liner of a gas turbine engine. Specifically, we demonstrate that this metamaterial results in components that are mechanically resilient throughout numerous thermo-mechanical loading cycles, provide efficient effusion cooling, and supply acoustic damping. The multifunctional performance for this family of metamaterial designs is experimentally characterized with testing of its fatigue, cooling effectiveness, and acoustic damping properties.

To begin our investigation of a suitable metamaterial for use in the combustor of a gas turbine engine, we start with the S-slot mechanical metamaterial that exhibits auxetic behavior with low-porosity ${ }^{[10]}$ and minimal stress concentrating features. ${ }^{[9]}$ When load is applied to this metamaterial, normal strains are coupled to the rotation of stiff domains that are located inbetween slots of the same orientation. This pattern of deformation closely resembles the mechanism of rotating squares ${ }^{[7]}$ that is known to have auxetic behavior. The auxetic property is beneficial for a combustor; in addition to the counter-intuitive lateral deformation of auxetic materials when subjected to uniaxial loads, negative Poisson's ratio implies that the bulk 


\section{WILEY-VCH}

modulus of the metamaterial is substantially smaller than the parent material it is composed of. Since combustors can be idealized as a constrained body that experiences thermal loading, the low bulk modulus of an auxetic material reduces the average stress it experiences. Although the mechanical properties of one such metamaterial design has been studied ${ }^{[9]}$, we are interested in the performance of this material for use in a combustor, requiring consideration of its cooling and acoustic damping characteristics as well. To maximize its suitability as a material for a combustor, we optimize a generalization of the S-slot material using the parameters shown in Figure 2a. Five parameters are used to define the geometry of the S-slot, and two parameters determine the spacing between adjacent slots. Members within this family of designs preserve the deformation pattern found in the starting design,${ }^{[9]}$ while facilitating the exploration of additional geometries that may be superior in the multifunctional context. To determine the mechanical performance of the S-slot material for use in a combustor, we perform finite element simulations on unit cells with periodic boundary conditions. We load the metamaterial by applying a small equibiaxial strain field, which is equivalent to the loading imposed by constrained thermal expansion. The same loading is applied to the standard circular hole design that is used as a benchmark for comparison. The stress contours for the circular hole and one of the S-slot materials are shown in Fig. 2b. Since the primary failure mode for a combustor is through the initiation and growth of fatigue cracks, the maximum value of Mises stress on the unit cell is of primary interest. Both of these materials have a porosity of 5\%, yet the S-slot exhibits a peak Mises stress that is less than half of the value in the benchmark case. Remarkably, the S-slot even achieves a peak stress that is less than the uniform stress that would be present in an unperforated sheet. This reduction in stress is attributed to the benefit of using an auxetic material in this loading configuration.

To determine the cooling effectiveness of the S-slot material, we perform computational fluid dynamics simulations on a plate of finite domain with four slots. A hot 


\section{WILEY-VCH}

gas flow is prescribed across one surface of the plate, with a higher pressure of cool gas present on the other surface. As the cool gas flows through the perforations in the plate, it forms a film that decreases the near wall gas temperature on the other surface of the plate. The same conditions are applied to a plate with circular holes that is used as a benchmark for comparison. The wall temperatures for the same circular hole and S-slot designs from Fig. 2b are shown in Fig. 2c. To assess the cooling performance of each design we use the cooling effectiveness $\eta_{\text {tot }}$ averaged over the domain. $\eta_{\text {tot }}$ is given by Equation 1:

$$
\eta_{\text {tot }}=\frac{T_{2}-T_{w}}{T_{2}-T_{1}}
$$

Where $T_{1}$ is the temperature of the cooling air, $T_{2}$ is the temperature of the hot gas, and $T_{w}$ is the temperature of the material. From these simulations, it is observed that the S-slot design spreads the cooling air more evenly across the wall surface, leading to an improved average cooling effectiveness $\eta_{\text {tot }}$

To find the best $S$-slot designs for the multifunctional requirements of a combustor, we search for the Pareto front with the two objectives of stress minimization and maximal cooling effectiveness. To constrain the absolute size of the S-slots during optimization, one of the geometric parameters is held constant, with $b=0.5$ To reduce the computational cost of optimization, the peak stress and cooling effectiveness from direct simulation are fit with surrogate models of the form given in Equation 2:

$$
y=C+\sum_{i=1}^{N} C_{i} x_{i}+\sum_{i=1}^{N} \sum_{j=1}^{N} C_{i j} x_{i} x_{j}
$$

where $\mathrm{y}$ is the response variable, $\mathrm{C}$ are the fitting coefficients, and $\mathrm{x}$ are the geometric parameters of the S-slot. The surrogate model for peak stress is fit to results from the stress analysis of $49 \mathrm{~S}$-slot designs, and the model for cooling effectiveness is fit to the results from 25 simulations of the cooling effectiveness. The Pointer algorithm ${ }^{[11]}$ is used to search the 


\section{WILEY-VCH}

surrogate models for the Pareto front, with satisfactory convergence occurring after sampling a few thousand points. To apply the S-slot material to a real combustor, the ability to spatially vary the material properties is necessary. For this purpose, the Pareto front is mapped for Sslot designs with porosity values of 3\%, 5\%, and $9 \%$. The stress reduction and enhancement of cooling effectiveness for designs on these Pareto fronts compared to the benchmark cases are shown in Fig. 2d. All S-slot designs on the Pareto front are found to substantially outperform the standard circular holes used in modern gas turbine combustors. For each value of porosity, the Pareto optimal S-slot designs all exhibit greater sensitivity of the peak stress to the geometry than the cooling effectiveness. For example, at 5\% porosity the variation in stress across the Pareto front is $40 \%$, while the variation in cooling effectiveness is just $7 \%$. To make use of the optimized S-slot materials in the combustor of a gas turbine engine the design must pass a variety of tests to assess its fatigue, cooling, and acoustic damping performance during testing conditions that most closely match those found a functioning gas turbine engine. We start with cyclic mechanical testing to compare the fatigue behavior between the S-slot material and the standard circular hole design. The fatigue specimens are shown in Fig. 3a and consist of a thin wall tube of Haynes 230 that has the cooling hole pattern laser cut into it. Testing with a tubular specimen is necessary to capture the effect that the curvature of a combustor will have on the fatigue life. Both the circular hole and S-slot specimens are tested at a porosity of $9 \%$, with the geometry of the S-slot selected to be similar to the design located at the center of the Pareto front. The geometry of the S-slot slightly differs from the Pareto optimal solution with the values of all the geometric parameters being: $\mathbf{a}=\mathbf{1 . 6}, \theta=90^{\circ}, \mathbf{s}_{\mathbf{x}}=\mathbf{s}_{\mathbf{y}}=\mathbf{5}, \mathbf{h}=\mathbf{2 . 7 5}$, and $\mathbf{t}=\mathbf{0 . 1 2}$. Isothermal fatigue tests (see $S I$ for an image of the test setup) are performed at $300^{\circ}, 600^{\circ}$, and $800^{\circ} \mathrm{C}$, corresponding to temperatures that are representative of the entry region, mid-section, and hottest spots in a combustor, respectively. Thermomechanical fatigue (TMF) tests (see Fig. $3 \mathrm{~b}$ for test setup) were also performed to simulate engine start-up/shutdown cycles. TMF testing is performed 


\section{WILEY-VCH}

with temperature oscillations that are in phase and out of phase with the strain oscillations to simulate the conditions experienced at the cold and hot sides of a combustor. Two temperature ranges are tested with TMF testing, $300-750^{\circ}$ and $300-850^{\circ}$. Both the isothermal fatigue and TMF testing were conducted under strain control in accordance to standard test practices defined in ASTM E606 and E2368, respectively.

The cycling data for a representative specimen with circular holes and S-slots are shown in Fig. 3c. The ability of the S-slot material to decrease the average stress in the material is apparent by the substantially smaller peak load it reaches during the strain controlled cycles. This decreases the energy available for crack growth, allowing the S-slot material to endure more than 10 times as many cycles as the standard circular hole design before crack initiation, which was defined to occur when a 10\% drop in the load was observed. The number of cycles required for crack initiation is shown for the standard circular and optimized S-slot designs in Figure 3d for the various test conditions. In all test conditions, the S-slot material substantially outperforms the standard design. The requirement to test these geometries in the various conditions experienced within a combustor is shown to be critical since the improvement of the S-slot material over the circular hole varies substantially across the testing conditions. For example, the fatigue life for isothermal cycling at $600^{\circ} \mathrm{C}$ is improved by 160 times, whereas the improvement for out of phase TMF up to $750^{\circ}$ is more modest at 4.6. Nonetheless, in commercial application where even incremental improvements are critical, the most conservative gain in fatigue life presented here with the S-slots over the standard design is a striking improvement.

To experimentally assess the cooling and acoustic performance of the S-slot material, planar specimens with S-slots and circular holes were fabricated from plates of Haynes 230. For both specimens, a tessellation of nine by 24 unit cells was laser cut, targeting a porosity of $3 \%$. Due to fabrication tolerances, the porosities were measured as $3.17 \%$ and $2.71 \%$ for the S-slot and circular hole specimens, respectively. The geometry of the S-slot is selected to be 


\section{WILEY-VCH}

similar to the design located at the center of the Pareto front, however the geometry of the Sslot slightly differs from the Pareto optimal solution with the values of the geometric parameters being: $\mathbf{a}=\mathbf{0 . 9}, \theta=90^{\circ}, \mathbf{s}_{\mathbf{x}}=\mathbf{s}_{\mathbf{y}}=\mathbf{6 . 4}, \mathrm{h}=3.0$, and $\mathrm{t}=0.12$. The effusive cooling and acoustic damping performance of the specimens were tested in a novel wind tunnel facility known as the Hot Acoustic Test rig (HAT) ${ }^{[12]}$, jointly operated by the German Aerospace Center and TU Berlin (see SI for details on the testing device). For these tests, a main flow of hot air grazes along one face of the test specimens, while cool air flows through the perforations in the test specimens. The cooling flow is maintained at $15^{\circ} \mathrm{C}$ and passes through a restrictive metering layer before reaching the test specimen. For each temperature of the hot flow tested, two conditions of the cooling flow are tested corresponding to pressure drops $\Delta \mathrm{P}$ of $1 \%$ and $3 \%$ between the cooling air supply to the metering layer and the hot flow.

For experiments to assess the cooling effectiveness of the test specimens, the hot flow is set to temperatures of $250^{\circ}$ and $400^{\circ} \mathrm{C}$. The temperature of the specimens is monitored with three K-type thermocouples (located at upstream, central, and downstream locations) and an infrared camera. Contour maps of the cooling effectiveness $\eta_{\text {tot }}$ near the center of the experimental specimens are shown in Figure 4a-b. It is observed that the cooling effectiveness of the specimen with S-slots outperforms that of the benchmark specimen. The cooling effectiveness $\eta_{\text {tot }}$ measured by the centrally located thermocouple for all four test conditions is shown in Figure 4c. The S-slot material is found to outperform the benchmark design for all cases of hot gas temperature and cooling flow pressure drop $\Delta \mathrm{P}$. Averaging across all conditions tested, the cooling effectiveness of the S-slot material is $21 \%$ better than that of the benchmark design.

To test the last critical property required for a combustor, acoustic damping, acoustic measurement are performed on the same two specimens used for the cooling tests. To measure the acoustic damping properties of the specimens, two speakers powered alternatively with multi-tone sine waves are located on either side of the specimen. Between 


\section{WILEY-VCH}

each speaker and the specimen, the signal measured by five microphones is used to calculate the reflection, transmission, and dissipation coefficients of the driving signal. The acoustic properties are measured with hot gas flows at both $250^{\circ}$ and $500^{\circ} \mathrm{C}$, and with cooling air pressure drops $\Delta \mathrm{P}$ of $1 \%$ and $3 \%$ at each temperature. The average of the upstream and downstream acoustic dissipation is shown for each specimen across a range of frequencies in Figure 4d. It should be noted that the peak in dissipation around $1200 \mathrm{~Hz}$ is largely influenced by a resonant mode of the cavity formed between the specimen and metering layer. Outside of this affected frequency range, the acoustic dissipation provided by each S-slot design is found to either match or outperform the benchmark design. At low frequencies, insensitivity of the

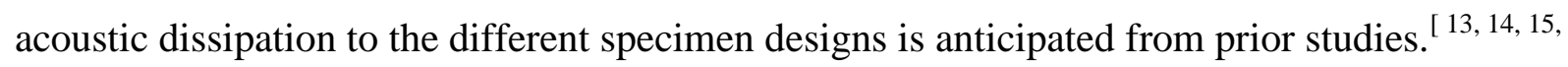
${ }^{16,17]}$ For frequencies about $2 \mathrm{kHz}$, the $\mathrm{S}$-slot material outperforms the benchmark design, likely due to increased viscous losses in the narrow slots.

From the perspective of achieving the multifunctionality required for a combustor, the S-slot material demonstrates a remarkable improvement over the typically used circular hole design. When the properties of fatigue life, cooling performance, and acoustic damping are considered separately, the S-slot material exhibits substantially better performance.

Furthermore, the coupling of these improvements brings about far more benefit than any one improvement. If applied to a modern gas turbine engine, the fatigue life of the combustor would not only be increased by the improved stress management from the S-slot material, the reduction in thermal strain from the enhanced cooling performance would extend the fatigue life significantly further. The synergistic improvement in performance from applying mechanical metamaterials to engineered systems that operate near the limit of their materials offers a disruptive avenue to achieve superior performance without the need for new materials. 


\section{WILEY-VCH}

\section{Supporting Information}

Supporting Information is available from the Wiley Online Library or from the author.

Received: ((will be filled in by the editorial staff))

Revised: ((will be filled in by the editorial staff))

Published online: ((will be filled in by the editorial staff))

\section{References}

[1] J. B. Pendry, A. J. Holden, D. J. Robbins, and W. Stewart, IEEE transactions on microwave theory and techniques, vol. 47, no. 11, pp. 2075-2084, 1999.

[2] R. Stevenson, M. Sazegar, A. Bily, M. Johnson, and N. Kundtz, Advanced Electromagnetic Materials in Microwaves and Optics (METAMATERIALS), 2016 10th International Congress on, pp. 349-351, IEEE, 2016.

[3] E. Brookner, "Metamaterial advances for radar and communications," 2017.

[4] A. Boyle, "Echodyne gets $\$ 29 \mathrm{~m}$ from nea, bill gates and others to boost radar for drones," Geek Wire.

[5] R. Lakes, Science, vol. 235, pp. 1038-1041, 1987.

[6] G. W. Milton, Journal of the Mechanics and Physics of Solids, vol. 40, no. 5, pp. 1105-1137, 1992.

[7] J. N. Grima and K. E. Evans, Journal of Materials Science Letters, vol. 19, no. 17, pp. $1563-1565,2000$.

[8] C. Tiedemann, D. Peitsch, and T. Schliwka, "Interaction of main flow and sealing air across a turbine cavity under unsteady conditions," ISABE 2015 (Awatef Hamed, Faculty Work and Research, College of Engineering and Applied Science), 2015.

[9] F. Javid, J. Liu, A. Rafsanjani, M. Schaenzer, M. Q. Pham, D. Backman, S. Yandt, M.C. Innes, C. Booth-Morrison, M. Gerendas, et al., Extreme Mechanics Letters, vol. 16, pp. $13-17,2017$. 


\section{WILEY-VCH}

[10] M. Taylor, L. Francesconi, M. Gerendás, A. Shanian, C. Carson, and K. Bertoldi, Advanced Materials, vol. 26, no. 15, pp. 2365-2370, 2014.

[11] A. Van der Velden and D. Kokan, ASME 2002 International Design Engineering Technical Conferences and Computers and Information in Engineering Conference, pp. 159165, American Society of Mechanical Engineers, 2002.

[12] C. Lahiri, K. Knobloch, F. Bake, and L. Enghardt 2013 “Acoustic measurements of perforated liners in hot and pressurized flow”. In: Proceedings of ASME Turbo Expo 2013 (GT2013-94674). ASME Turbo Expo 2013, June 3-7, 2013, San Antonio, Texas, USA.

[13] U. Ingard, The Journal of the acoustical society of America, vol. 25, no. 6, pp. 10371061, 1953.

[14] A. Dowling and I. Hughes, Journal of Sound and Vibration, vol. 156, no. 3, pp. 387405, 1992.

[15] S. Grace, K. Horan, and M. Howe, Journal of fluids and structures, vol. 12, no. 3, pp. $335-351,1998$.

[16] C. Lahiri, L. Enghardt, F. Bake, S. Sadig, and M. Gerendás, Journal of Engineering for Gas Turbines and Power, vol. 133, no. 9, p. 091503, 2011.

[17] F.-X. Jetté, A. Shanian, M. Schaenzer, M. Q. Pham, G. Bourgeois, M. Farhangi, F. Sanchez, and M. Innes, ASME Turbo Expo 2018: Turbomachinery Technical Conference and Exposition, pp. V04AT04A061-V04AT04A061, American Society of Mechanical Engineers, 2018. 


\section{WILEY-VCH}

\section{Functionality}

Effusive cooling Stress management Acoustic damping
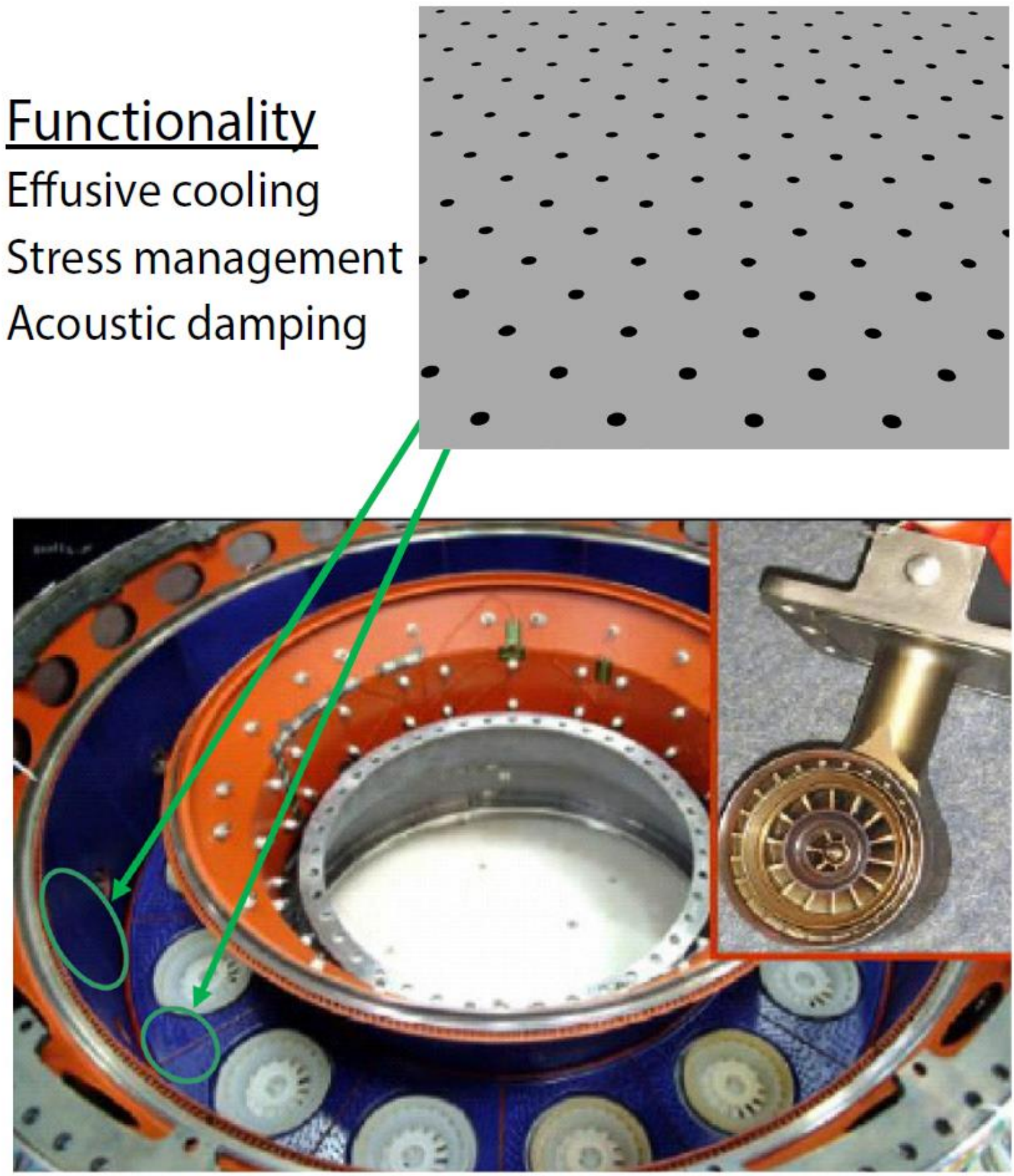

Figure 1. A prototypical annular combustor with a magnified rendering of the perforated material used in part of its construction. The perforated material permits the flow of cooling air through the material to prevent it from overheating and provide acoustic dissipation to the flow while managing thermally induced stresses to prevent fatigue failure. 


\section{WILEY-VCH}

(a)

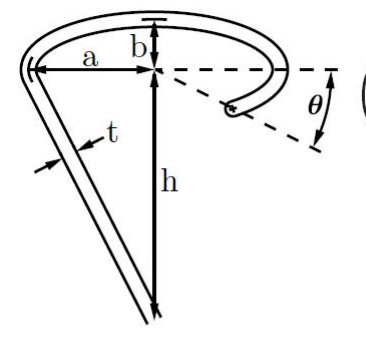

(c)

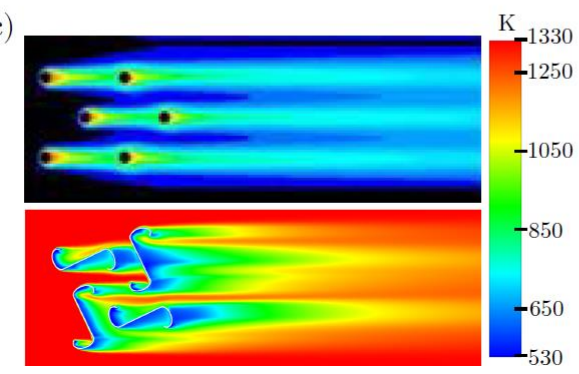

(b)

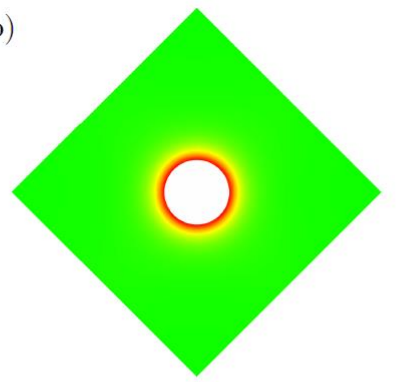

(d)

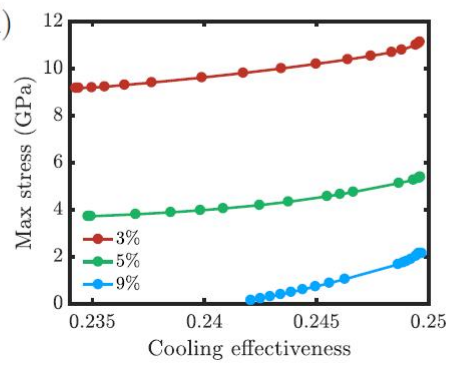

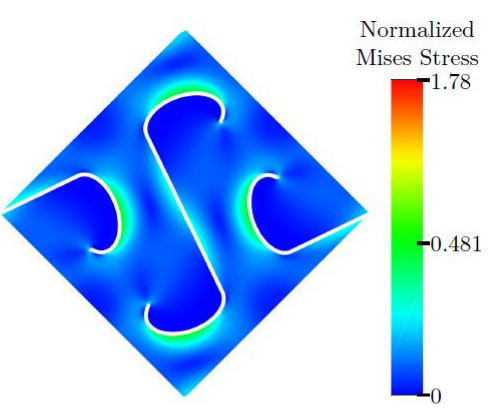

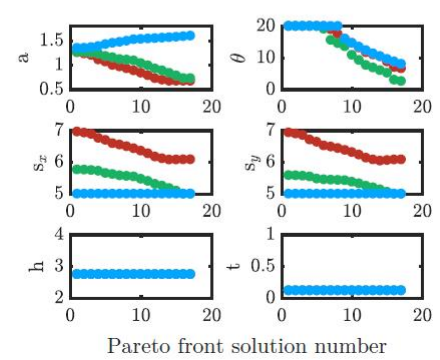

Figure 2. (a) The six geometric parameters that define the S-slot metamaterial. During optimization, the parameter $\mathrm{b}$ is held constant. (b) One of the mechanical simulations from the optimization of S-slot geometry compared to the conventional design of circular holes. Both are subjected to the same level of equibiaxial strain with periodic boundary conditions. Contours of von Mises stress are normalized by the value of stress present in an unperforated plate loaded in the same manner. (c) One of the fluid flow simulations from the optimization of the S-slot geometry compared to the conventional design. Contours correspond to the near wall gas temperatures. (d) Pareto fronts from the multi-objective optimization at three values of porosities, and the values of the S-slot geometric parameters for each solution on the Pareto front. 


\section{WILEY-VCH}

(a)
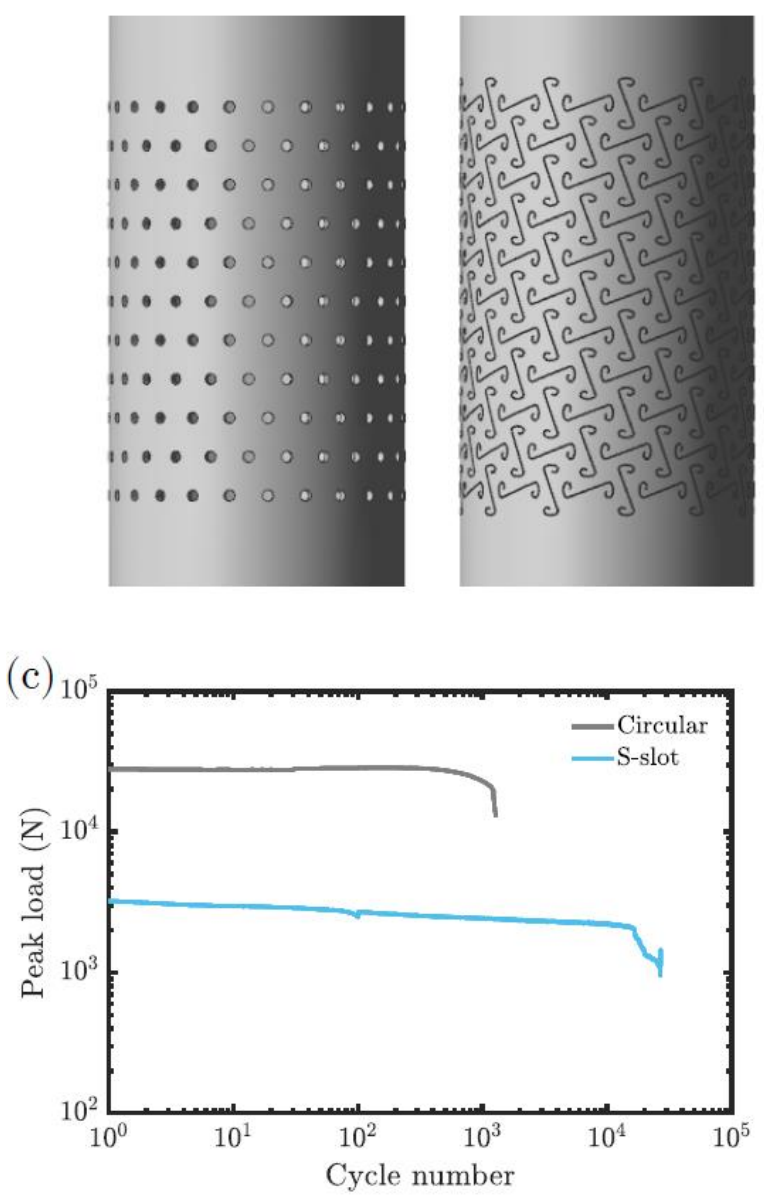

(b)
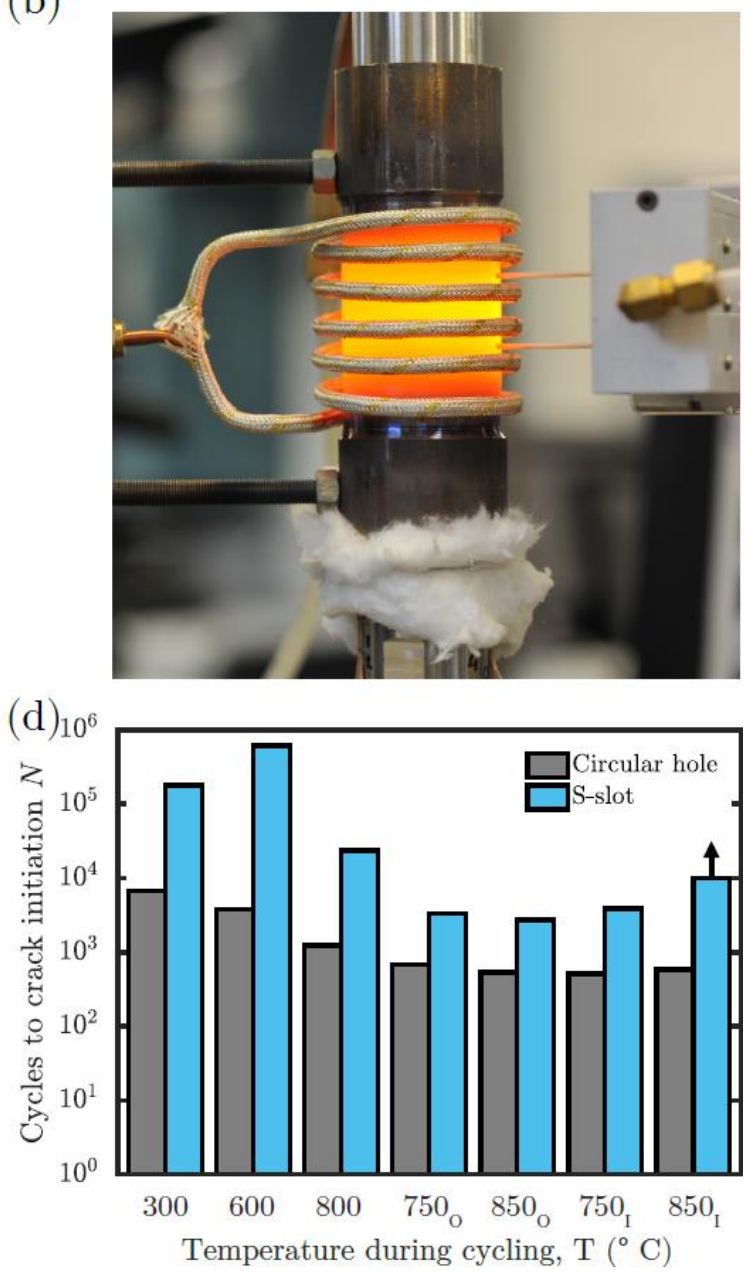

Figure 3. (a) Tubular specimen designs for fatigue testing of the circular hole geometry and one of the S-slot geometries. (b) Thermo-mechanical fatigue testing in progress. Primary components of the setup include induction coils, an Inconel susceptor around the specimen, a high temperature extensometer, forced air cooling of the specimen, and a load cell (located outside the field of view of the image). (c) Typical mechanical behavior during cyclic loading from isothermal fatigue of a circular hole and S-slot specimen. (d) Summary of all fatigue testing. Temperatures with a subscripts "O" and "I" are tested in out of phase and in phase TMF, respectively. TMF tests are labeled according to the maximum temperature applied in the thermal cycle. The arrow on the S-slot bar labeled 850I indicates that the specimen did not initiate fatigue cracks after 10,000 cycles and further testing was discontinued. 
WILEY-VCH

(a)

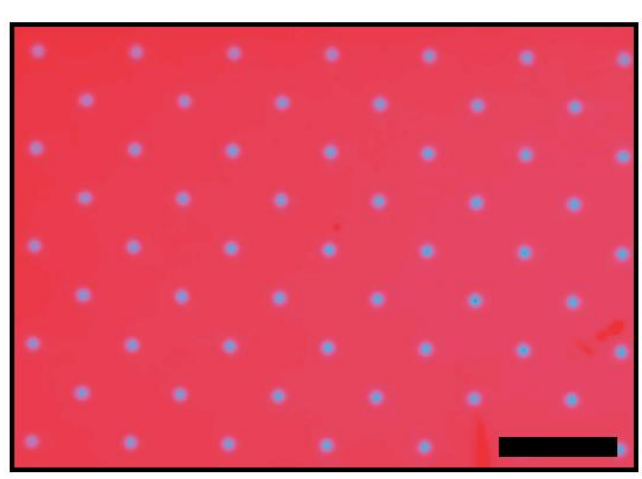

(c)

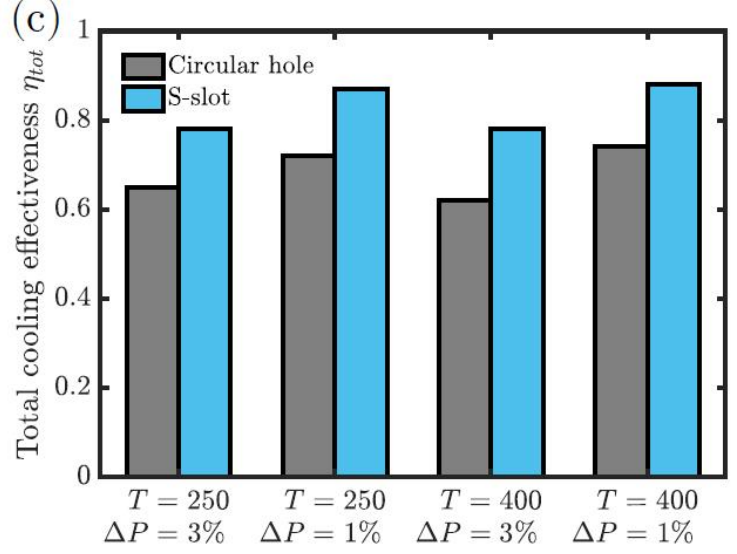

(b)

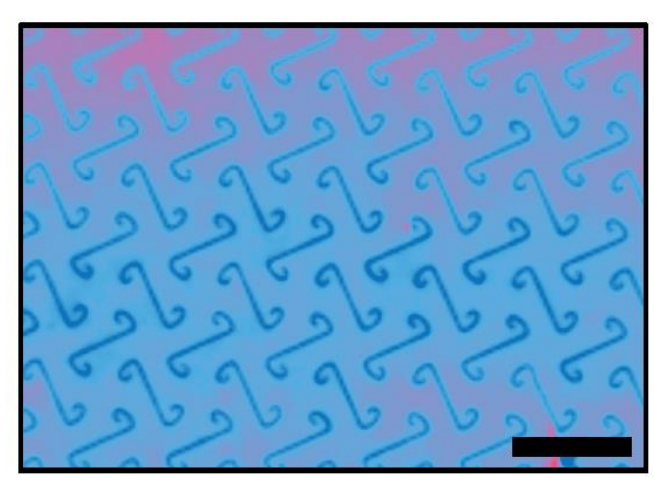

(d)

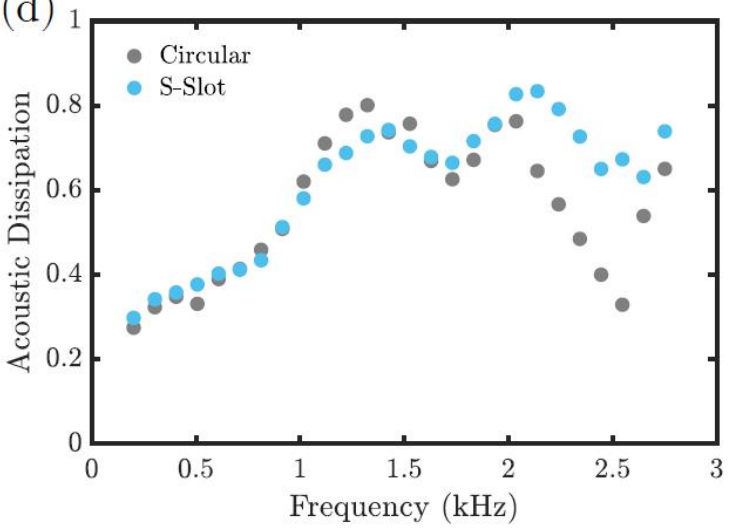

Figure 4. (a) Local cooling effectiveness as measured from thermal imaging of the wall temperature on the circular hole cooling specimen. Scale bar is $10 \mathrm{~mm}$. (b) Local cooling effectiveness as measured from thermal imaging of the wall temperature on the S-slot cooling specimen. Scale bar is $10 \mathrm{~mm}$. (c) Summary of cooling performance across different flow and temperature conditions. (d) Typical acoustic damping performance of the circular hole and Sslot specimens. Data in (a), (b), and (d) are taken from experiments with a hot flow temperature $\mathrm{T}=250 \circ \mathrm{C}$ and pressure drop $\Delta \mathrm{P}=1 \%$ 


\section{WILEY-VCH}

Table of Contents

This paper describes the application of auxetic materials for use in high temperature combustion applications. The results of our findings show that our optimized auxetic multifunctional metamaterial outperforms conventional designs for cooling systems commonly used in high temperature industrial applications.

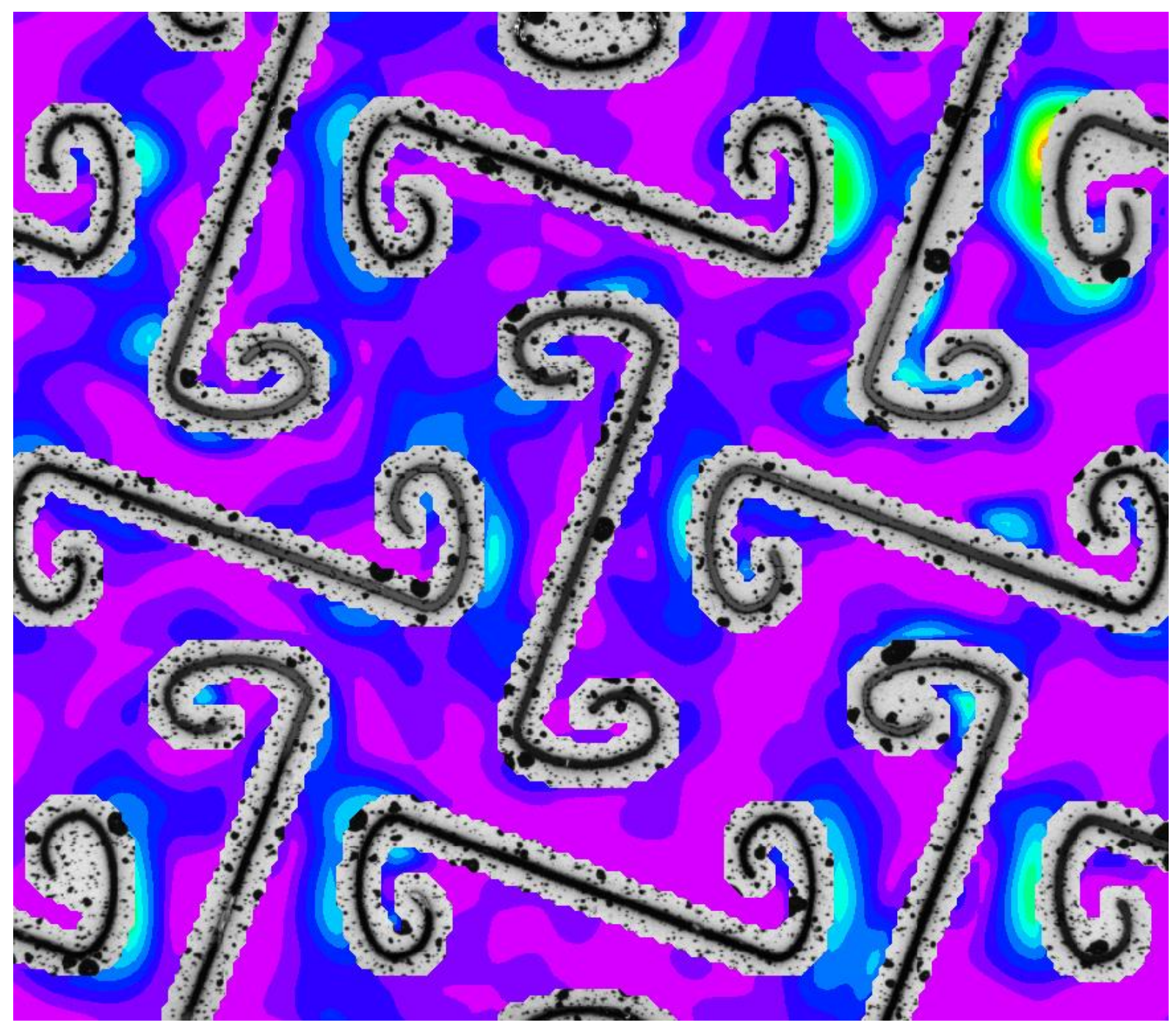

